\title{
Trans-presentation of interleukin-15 by interleukin-15 receptor alpha is dispensable for the pathogenesis of autoimmune type 1 diabetes
}

\author{
Diwakar Bobbala ${ }^{1}$, Marian Mayhue ${ }^{1}$, Alfredo Menendez ${ }^{2,3}$, Subburaj Ilangumaran $^{1,3}$ \\ and Sheela Ramanathan ${ }^{1,3}$
}

Interleukin-15 (IL-15) is a pro-inflammatory cytokine that is required for the survival and activation of memory $\mathrm{CD} 8^{+} \mathrm{T}$ cells, natural killer (NK) cells, innate lymphoid cells, macrophages and dendritic cells. IL-15 is implicated in the pathogenesis of various autoimmune diseases such as rheumatoid arthritis, inflammatory bowel disease, psoriasis and autoimmune type 1 diabetes (T1D). IL-15 receptor (IL-15R) consists of a specific $\alpha$ chain, the $\beta$ chain that is shared with IL-2R and the common $\gamma$ chain. IL-15 is unique in the manner in which it binds and signals through its receptor subunits. IL-15 that is complexed with IL-15R $\alpha$ binds to the $\beta \gamma$ receptor complex present on the responding cell to mediate its biological effects through a process referred to as trans-presentation. The trans-presented IL-15 is essential to mediate the biological effects on T lymphocytes and NK cells. Here we show that IL-15, but not IL-15R $\alpha$, is required for the development of spontaneous and virus-induced T1D, viral clearance and for antigen cross-presentation to CD8 ${ }^{+}$T lymphocytes. Our findings provide insight into the complexities of IL-15 signalling in the initiation and maintenance of $\mathrm{CD}^{+} \mathrm{T}$ cell-mediated immune responses.

Cellular \& Molecular Immunology (2017) 14, 590-596; doi:10.1038/cmi.2015.102; published online 8 February 2016

Keywords: cross-presentation; IL-15 trans-presentation; lymphocytic choriomeningitis virus; type 1 diabetes

\section{INTRODUCTION}

Interleukin-15 (IL-15) plays a multifaceted role in innate and adaptive immunity by regulating the development, homeostasis and functions of hematopoietic and non-hematopoietic cells. ${ }^{1,2}$ Even though IL-15 mRNA can be detected in different organs such as placenta, skeletal muscle, intestine and kidney under steady state, ${ }^{3}$ the protein expression is below the level of detection by conventional methods and is limited to macrophages and dendritic cells (DCs). ${ }^{4}$ IL-15 protein can be expressed with either a short or a long signal peptide (SSP and LSP, respectively). Whereas the SSP-IL-15 is not released from cells, the LSP-IL-15 is inefficiently secreted. ${ }^{5}$

The IL-15 receptor (IL-15R) complex consists of the ligandbinding IL-15R $\alpha$ chain (CD215), the $\beta$ chain (CD122; also used by IL-2) and the common gamma $\left(\gamma_{c}\right)$ chain. $^{2}$ The biological activities of IL-15 are mostly mediated by the IL-15:IL-15R $\alpha$ complex, produced by non-T cells and 'trans-presented' to responder cells that express the IL-2/15R $\beta \gamma_{c}$ complex. ${ }^{6}$ This peculiar and almost unique requirement for trans-presentation by IL-15R $\alpha$ suggests that the biological effects of IL-15 are tightly regulated not only at the level of IL- 15 production but also by the constraints imposed by trans-presentation. Tissuespecific ablation of IL- $15 R \alpha$ has revealed distinct patterns of requirement for the source of the trans-presented IL-15. For example, natural killer (NK) cells require IL-15 to be trans-presented by both DCs and macrophages while central memory $\mathrm{CD} 8{ }^{+} \mathrm{T}$ cells require DC-derived IL- $15 .{ }^{7,8}$ Moreover, cell-bound IL-15 can act in cis on producer cells ${ }^{9}$ and the IL-15: IL-15R $\alpha$ complex can be released by proteolytic cleavage, ${ }^{10}$ indicating further complexity in IL-15 signalling.

While studies using mice deficient for either IL-15 or IL$15 \mathrm{R} \alpha$ have clearly established that both are required for the development and homeostasis of NK cells and $\mathrm{CD}^{+} \mathrm{T}$ cells, deciphering the role of IL-15R $\alpha$ in immune responses remains a challenging issue due to the complexities of IL-15 signalling outlined above. ${ }^{11}$ IL-15 plays an important role in anti-viral immune responses, both in activating the innate immune system and in generating effective memory. ${ }^{12-14}$ IL-15 also

${ }^{1}$ Immunology Division, Department of Pediatrics, Université de Sherbrooke, Sherbrooke, QC, Canada; ${ }^{2}$ Department of Microbiology, Faculty of Medicine and Health Sciences, Université de Sherbrooke, Sherbrooke, QC, Canada and ${ }^{3}$ Centre de rechercheclinique, Université de Sherbrooke, Sherbrooke, QC, Canada Correspondence: S Ramanathan, PhD, Immunology Division, Department of Pediatrics, Faculty of Medicine and Health Sciences, Université de Sherbrooke, 3001 North 12th Avenue, Sherbrooke, QC J1H 5N4, Canada. Email: Sheela.Ramanathan@Usherbrooke.ca

Received: 23 September 2015; Revised: 15 November 2015; Accepted: 15 November 2015 
contributes to the pathogenesis of several autoimmune diseases including inflammatory bowel disease, rheumatoid arthritis and psoriasis. ${ }^{15}$ Recently, we and others have shown that IL15 plays a crucial role in the pathogenesis of autoimmune type 1 diabetes (T1D). ${ }^{16,17}$ In this study, we investigated whether IL15 trans-presentation plays an essential role in T1D pathogenesis. We show that IL-15R $\alpha$ deficiency does not affect T1D incidence in two different mouse models and that IL-15R $\alpha$ is also dispensable for antigen cross-presentation to $\mathrm{CD} 8^{+} \mathrm{T}$ cells, and key process in activating autoreactive $\mathrm{CD} 8^{+} \mathrm{T}$ cells.

\section{MATERIALS AND METHODS}

\section{Mouse strains}

NOD.Il15 ${ }^{-1}$ and NOD.scid.Il15 ${ }^{-/}$mice have been previously described. ${ }^{16} \mathrm{Il}_{15 \mathrm{ra}^{-1}}$ mice obtained from the Jackson laboratory were backcrossed to $\mathrm{C} 57 \mathrm{Bl} / 6$ and non-obese diabetic (NOD) mice for 6 and 11 generations, respectively. NOD.scid mice were obtained from the Jackson laboratory and crossed with $\mathrm{Il}_{15 \mathrm{ra}^{-/-} \text {mice to generate NOD.scid.Il15ra }}{ }^{-1-}$ mice. Rat insulin promoter-glycoprotein (RIP-GP) transgenic mouse expressing the envelope GP antigen of lymphocytic choriomeningitis virus (LCMV) in pancreatic islets ${ }^{18,19}$ were crossed with $I l 15^{-1}$ and $I l 15 \mathrm{ra}^{-/-}$mice to generate RIP-GP.Il $15^{-/}$and RIP-GP.Il15 $\mathrm{ra}^{-/-}$mice. RIP-GP.Rag $1^{-/-}$mice were generated in our animal facility. ${ }^{20}$ OT-1 TCR transgenic mice were obtained from Jackson laboratories and were crossed with $\mathrm{C} 57 \mathrm{Bl} / 6$. $\mathrm{Il}_{15 \mathrm{ra}^{-/}}$mice to generate OT-1.Il15 $\mathrm{ra}^{-/-}$mice. Mice were housed in micro-isolated sterile cages under specific pathogen-free conditions. All experimental protocols on mice were approved by Université de Sherbrooke Ethics Committee for Animal Care and Use in accordance with guidelines established by the Canadian Council on Animal Care.

\section{LCMV and Listeria strains}

LCMV Armstrong strain and Listeria monocytogenes expressing ovalbumin protein (LM-OVA) were kind gifts from Drs P. Ohashi (University of Toronto) and C. Krawczyk (McGill University), respectively. LCMV stock was prepared through a single passage in BHK21 cells. LM-OVA was grown in brain-heart infusion medium for $15 \mathrm{~h}$ at $37^{\circ} \mathrm{C}$.

\section{LCMV infections and adoptive transfer}

Eight-week-old RIP-GP, RIP-GP.Il15 ${ }^{-1}$ and RIP-GP.Il15 $\mathrm{ra}^{-/-}$mice were infected with $2 \times 10^{4}$ plaque forming units ( $\mathrm{pfu}$ ) of LCMV intravenously and monitored for T1D and insulitis. Splenocytes were isolated 14 days after infection of WT, $I l 15^{-/}$and $I l 15 \mathrm{ra}^{-/}$ mice and $1 \times 10^{7}$ cells were adoptively transferred to RIP-GP. $\mathrm{Ragl}^{-/-}$mice. The recipients were followed for T1D and insulitis.

\section{Monitoring T1D and evaluation of insulitis}

Spontaneous T1D development in NOD and NON.Il15ra ${ }^{-1-}$ mice were monitored for up to 40 weeks, using DIASTIX strips (Bayer, Leverkusen, Germany) to test the glucose in the urine. Mice were monitored three times a week. Mice were considered diabetic when three consecutive readings over a week were positive ( $>28 \mathrm{mM}$ in the urine). As the progression of T1D is rapid in the RIP-GP model of T1D, blood glucose levels were monitored every 2 days from day 7 after LCMV infection, and 5 days after adoptive transfer. T1D incidence was monitored in the blood using Accu-Check strips (Roche Diagnostics, Missisauga Canada). Animals that showed more than $15 \mathrm{mM}$ of blood glucose on two consecutive readings were considered diabetic and were euthanized. Insulitis was scored as previously described. ${ }^{16}$ Briefly, at killing, pancreases were fixed in buffered formalin and embedded in paraffin. Three non-overlapping (200 $\mu \mathrm{m}$ apart), 5- $\mu \mathrm{m}$ sections were stained with hematoxylin and eosin and blindly scored for insulitis.

\section{Viral clearance}

Viral titer was evaluated on day 14 following LCMV infection. The liver tissue was homogenized in phosphate-buffered saline using a bead mill MM 400 (Retsch, Hann, Germany). Serial 10fold dilution of the homogenate was tested on the fibrosarcoma cell line MC57G to calculate the plaque formation units, following published methods. ${ }^{21}$

\section{In vivo cross-presentation assay}

Mice of the indicated genotypes were infected with $5 \times 10^{3}$ colony forming units (CFU) of Listeria monocytogenes expressing ovalbumin protein (LM-OVA) intravenously on day 0 . The following day $10^{7} 5$-(6) carboxyfluorescein diacetate succinimidyl ester (CFSE) labelled CFSE-labelled splenocytes from OT1 or OT-1.Il15 $\mathrm{ra}^{-1-}$ mice were injected intravenously. ${ }^{22}$ Six days later, proliferation of CFSE-labelled OT-1 cells in the spleen was assessed by flow cytometry.

\section{Statistical analysis}

Cumulative incidence of T1D was analysed using the Prism software (GraphPad, California, USA). For diabetes incidence, statistical significance was calculated using log-rank (MantelCox) test.

\section{RESULTS AND DISCUSSION}

IL-15R $\alpha$ is dispensable for spontaneous T1D in NOD mice, but shows variable requirement for the maintenance of autoreactive $\mathrm{CD8}^{+} \mathrm{T}$ cells

We have shown previously that IL-15 plays a critical role in activating diabetogenic $\mathrm{CD}^{+} \mathrm{T}$ cells in the NOD mouse model. ${ }^{16}$ To determine whether trans-presentation of IL-15 is essential to activate pathogenic $\mathrm{CD}^{+} \mathrm{T}$ cells in T1D, we generated NOD.Il15 $\mathrm{ra}^{-/}$mice and followed them for T1D development. We observed that the cumulative incidence of T1D in female NOD.Il15 $\mathrm{ra}^{-/-}$mice was comparable to that of control NOD mice (Figure 1a), suggesting that IL-15R $\alpha$ is dispensable for IL-15-dependent pathogenic mechanisms in T1D.

It has been demonstrated that the lack of either IL-15 or IL$15 \mathrm{R} \alpha$ causes a decrease in the frequency of memory $\mathrm{CD} 8^{+} \mathrm{T}$ cells, ${ }^{1,23}$ and that IL-15 is required mainly for the maintenance of antigen-experienced memory $\mathrm{CD}^{+} \mathrm{T}$ cells and not for their generation. ${ }^{12,24}$ We have shown previously that $\mathrm{CD} 8^{+} \mathrm{T}$ cells from diabetic 8.3-NOD mice expressing a highly pathogenic transgenic TCR on $\mathrm{CD}^{+} \mathrm{T}$ cells caused T1D following adoptive transfer 
a
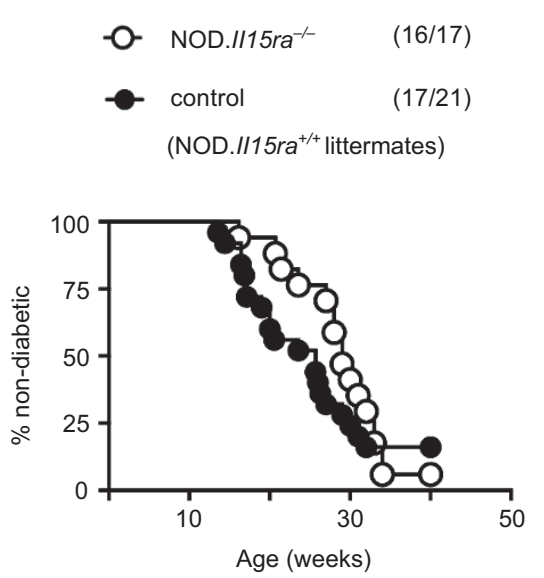

C

Donors: Diabetic NOD

Recipients (NOD.Scid) :

$\begin{array}{ll}- \text { + +/+ } & (7 / 8) \\ \text { - ㅇ } 1115 \mathrm{ra}^{-/-} & (10 / 12) \\ \text { ㅁ. } 1 / 15^{--} & (0 / 4)\end{array}$

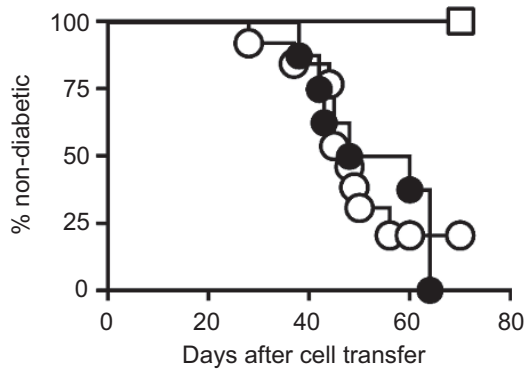

b

Donor : 8.3-NOD

Recipients (NOD.Scid) :

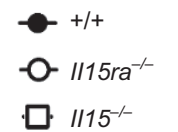

$5 / 5)$

$(0 / 6)$

$(0 / 5)$

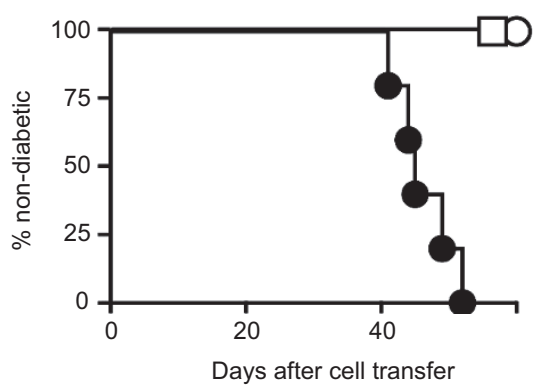

d

Donors: Diabetic NOD./115ra ${ }^{-/}$

Recipients (NOD.Scid) :
$\rightarrow+/+$
$(9 / 9)$
-O $1115 \mathrm{ra}^{-/}$
(0/10)

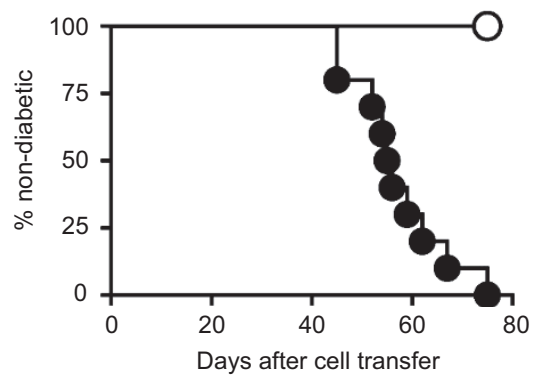

e Recipients (NOD.Scid) :

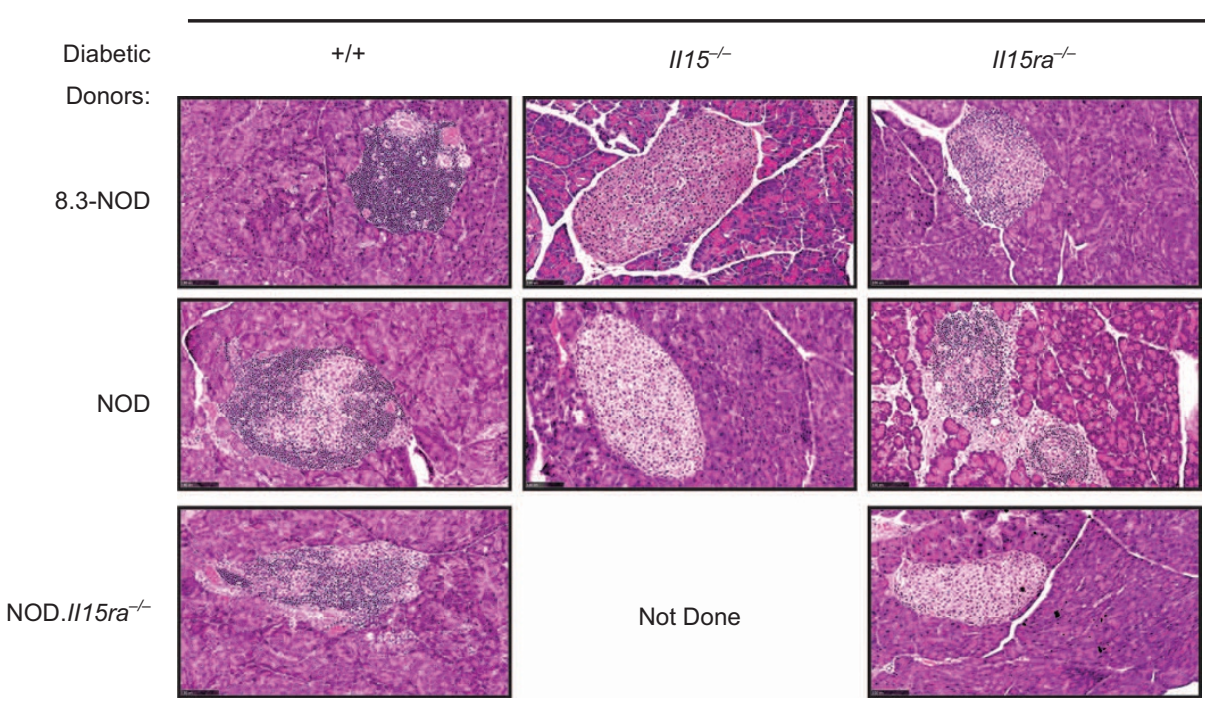

Figure 1 Spontaneous T1D development in NOD mice does not require IL-15R $\alpha$. (a) Cumulative incidence of T1D in female NOD. //15ra ${ }^{-/}$mice and control NOD. $/ 115 \mathrm{ra}^{+/+}$littermates is shown. The number of mice that developed diabetes over the number of infected mice is given in parenthesis. (b) Splenocytes $\left(1 \times 10^{7}\right.$ cells) pooled from female diabetic 8.3-NOD mice were adoptively transferred to 4-week-old male NOD.scid, NOD.scid. $/ / 15 \mathrm{ra}^{-1-}$ or $^{-}$ NOD.scid. $1 / 15^{-1}$ recipients. Data were pooled from two independent experiments. (c and d) Splenocytes ( $10^{7}$ cells) pooled from female diabetic NOD (c) or NOD. $/ / 15 \mathrm{ra}^{-1}$ (d) mice were adoptively transferred to 4-week-old male NOD.scid, NOD.scid.//15ra ${ }^{-1}$ or NOD.scid. $/ / 15^{-1-}$ (in c) recipients. Data pooled from three independent experiments are shown. (e) Pancreatic sections obtained at the time of killing were labelled with $\mathrm{H} \& \mathrm{E}$ for the detection of insulitis. 
in IL-15-sufficient NOD.scid mice but not in NOD.scid.Il15 $1-$ mice, ${ }^{16}$ indicating the requirement for IL-15 to sustain the diabetogenic potential of antigen-stimulated $\mathrm{CD}^{+} \mathrm{T}$ cells. Similarly, splenocytes from diabetic 8.3-NOD mice failed to induced T1D in NOD.scid.Il15ra ${ }^{-/-}$(Figure 1b), indicating that even though IL-15R $\alpha$ is dispensable for inducing T1D, antigen-experienced diabetogenic $\mathrm{CD}^{+}{ }^{+} \mathrm{T}$ cells require IL-15R $\alpha$ to sustain their autoimmune potential. However, islets of the recipient NOD.scid. $I l 15 \mathrm{ra}^{-/}$showed certain degree of insulitis (Figure 1e, top panel), suggesting that the injected cells had homed to the islets.

To assess if the results obtained with $8.3 \mathrm{TCR}$ transgenic $\mathrm{CD} 8{ }^{+}$ $\mathrm{T}$ cells also apply to polyclonal $\mathrm{CD} 8^{+} \mathrm{T}$ cell effectors, we adoptively transferred total splenocytes from diabetic NOD mice to NOD.scid recipients lacking IL-15 or IL-15R $\alpha$. While the donor cells failed to induce T1D in NOD.scid.Il15 ${ }^{1-}$ recipients, ${ }^{16}$ surprisingly NOD.scid.Ill5ra ${ }^{-/-}$recipients developed T1D with frequency and kinetics similar to that of control NOD.scid recipients (Figure 1c and e, middle panel). These results indicated that, contrary to the results obtained with $8.3 \mathrm{~T}$ cells, isletspecific effector $\mathrm{T}$ cells present in a polyclonal repertoire can be sustained even in the absence of trans-presented IL-15R $\alpha$.

It has been shown that the excess of soluble IL- 15 promotes the activation of $\mathrm{NK}$ and memory $\mathrm{CD}^{+}$subsets in mice and humans. ${ }^{6,25,26}$ Therefore, it is possible that IL-15 presents in the circulation of NOD.scid.Il15ra ${ }^{-/-}$recipients can bind to IL-15R $\alpha$ present on donor cells and trans-present IL-15 to autoreactive effector $\mathrm{CD}^{+}{ }^{+} \mathrm{T}$ cells. To address the role of donor-derived IL$15 \mathrm{R} \alpha$, we adoptively transferred splenocytes from diabetic NOD. $\mathrm{Il}_{15 \mathrm{ra}^{-/}}$mice to NOD.scid.Il15ra ${ }^{-/-}$recipients (Figure $1 \mathrm{~d}$ and e). T1D was not observed in the NOD.scid.Il15ra ${ }^{-1}$ recipients of splenocytes from diabetic NOD.Il15ra ${ }^{-/-}$mice, presumably due to the inability of the donor effector cells to be maintained in the absence of IL-15R $\alpha$ of either recipient or donor origin (Figure 1d). These mice showed minimal evidence for insulitis (Figure 1e, bottom panel). However, the inability of antigen-experienced 8.3 TCR transgenic $\mathrm{CD} 8^{+} \mathrm{T}$ cells to maintain their pathogenicity in NOD.scid.Il15 $\mathrm{ra}^{-/-}$recipients despite the availability of IL-15R $\alpha$ on donor cells (Figure 1b) begs additional explanation. It is possible that some of the autoreactive TCRs in the polyclonal repertoire may have overcome the dependency on IL-15R $\alpha$ due to higher affinity of their TCR toward their cognate autoantigens and/or help from donor $\mathrm{CD}^{+}{ }^{+} \mathrm{T}$ cells present in the polyclonal repertoire. ${ }^{27}$ Nevertheless, the above results indicate that (i) IL-15 trans-presentation is dispensable for the initial pathogenic activation of diabetogenic $\mathrm{T}$ cells, (ii) its requirement for sustaining the pathogenic potential of antigen-experienced $\mathrm{CD} 8{ }^{+} \mathrm{T}$ cells may vary depending on the clonal diversity of the autoreactive $\mathrm{T}$ cells, and (iii) when required for the latter, IL-15R $\alpha$ expressed on antigen-experienced $\mathrm{CD}^{+}{ }^{+} \mathrm{T}$ cells likely promotes their homeostatic expansion and/or pathogenic functions.

\section{IL-15R $\alpha$ is not needed for virus-induced T1D in the RIP-GP model and for clearing LCMV}

To further study the role of IL-15R $\alpha$ in the autoimmune disease process, we used the LCMV-induced model of T1D in the C57Bl/6 genetic background. RIP-GP mice express the LCMV
GP under the control of the rat insulin promoter. T cells specific to the GP antigen in the naive repertoire are ignorant of the transgenic viral protein expressed in pancreatic islets. ${ }^{18}$ Following LCMV infection, activated GP-specific cells recognize and destroy the islets resulting in T1D. ${ }^{18,28}$ RIP-GP transgenic control, Ill15 ${ }^{-1}$ and $\mathrm{Ill}_{\mathrm{Hra}} \mathrm{ra}^{-/}$mice were infected intravenously with $2 \times 10^{4}$ pfu of LCMV and were followed for T1D development. Even though T1D onset was delayed by 4-5 days in RIP-GP.Il15 $\mathrm{ra}^{-/}$mice when compared to control RIP-GP mice, 9 out of 10 mice developed T1D (Figure 2a). RIP-GP.Il15 ${ }^{-1}$ mice showed a similarly delayed disease onset; however, only $50 \%$ of them developed T1D even after 35 days. Consistent with the delayed disease kinetics RIP-GP.Il15ra ${ }^{-/}$ mice and RIP-GP.Ill $15^{/-}$mice that developed T1D showed less severe degree of insulitis, while control mice showed severe islet damage (Figure 2b). RIP-GP. $I l 15^{-1-}$ mice that did not develop T1D harboured islets that were mostly normal. Intriguingly, none of $I l 15^{/-}$RIP-GP mice had cleared the viral load at killing,
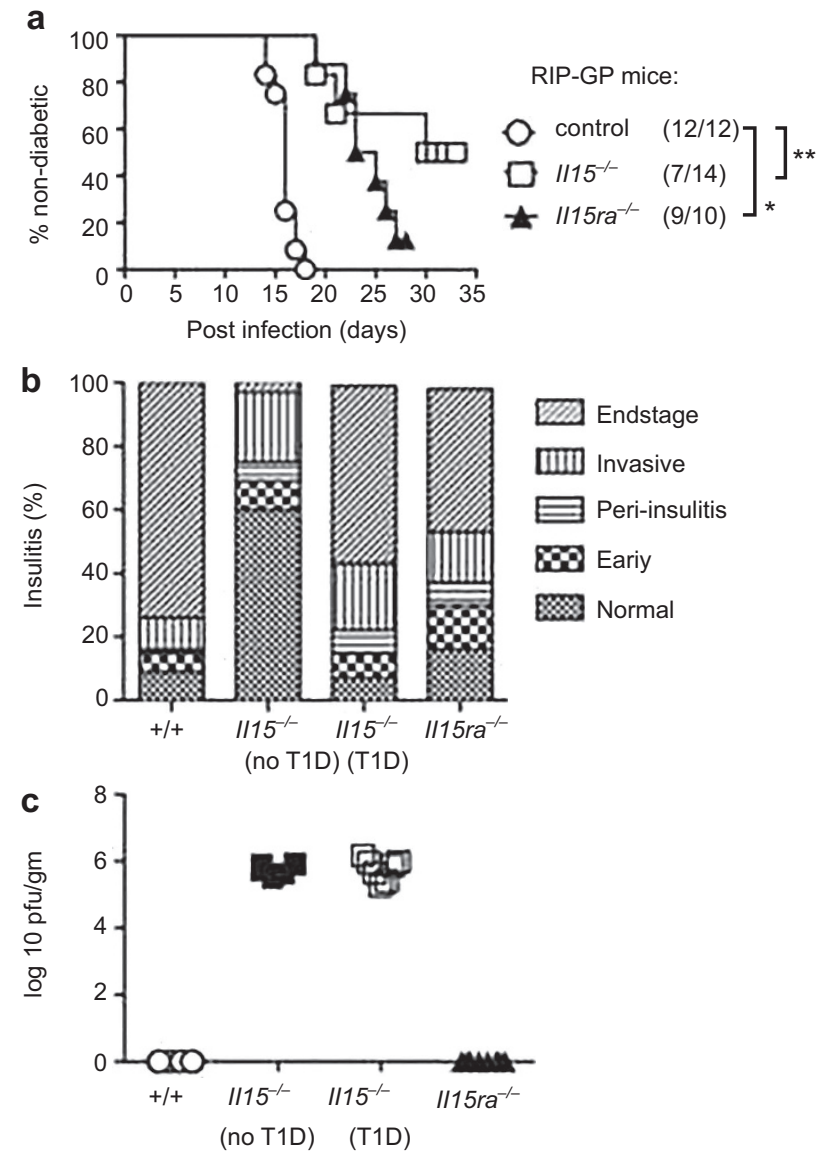

Figure 2 T1D induction by LCMV in RIP-GP mice is not affected by IL$15 R \alpha$ deficiency. (a) RIP-GP, RIP-GP. $/ / 15^{-1-}$ and RIP-GP. $/ / 15 \mathrm{ra}^{-1-}$ mice were infected with LCMV and T1D development was monitored. Log-rank test: $* P<0.05$; ** $P<0.01$. (b) Pancreatic sections obtained at the time of killing were scored for islet infiltration. (c) On day 14 post-infection, viral counts in the livers of infected mice were quantified by plaque forming assay on MC57G cells. Viral stock used for infecting the mice served as positive control (not shown). Data on cumulative incidence of T1D, insulitis and viral titer were pooled from three independent experiments. 
while RIP-GP.Il15ra ${ }^{-/-}$mice had efficiently cleared the virus (Figure 2c). As islet destruction in the RIP-GP model requires the induction of effector $\mathrm{CD} 8^{+} \mathrm{T}$ cell response again the transgenic GP antigen expressed in the islets, the above results indicate that the generation of functional, LCMV-specific effector T cells that can destroy the islets and clear the viral load do not require IL-15R $\alpha$. On the other hand, the indispensability of IL15 to clear LCMV and the variable requirement of IL-15 to induce T1D indicate that IL-15 is essential to generate protective antiviral responses, while activation of $\mathrm{CD} 8{ }^{+} \mathrm{T}$ cells specific to dominant viral epitopes may occur independently of IL-15.

A previous study has reported efficient clearance of LCMV in the absence of IL- 15 or IL-15R $\alpha^{13}$ The differences between the results presented here and the above study may result from the route of infection and the time from infection to the analysis of the viral load. Furthermore, viral clearance following systemic, but not mucosal route of infection with influenza virus has been shown to be dependent on IL-15. ${ }^{29}$

\section{IL-15R $\alpha$ is required to maintain effector $\mathrm{CD8}^{+} \mathrm{T}$ cells generated following viral infection}

The requirement for IL-15 during recall responses appears to vary depending on the pathogen involved as well as the route of infection. ${ }^{30,31}$ While IL-15 was shown to be necessary for memory generation following acute intravenous infections, ${ }^{12}$ memory $\mathrm{T}$ cells generated following the entry of pathogens via mucosal surfaces were not dependent on IL-15. ${ }^{14,29}$ To assess whether antigen-specific $\mathrm{T}$ cells that were generated in the absence of IL-15 trans-presentation retain their pathogenic potential, splenocytes from LCMV-infected control C57BL/6, $I l 15^{-1}$ and $I l 15 \mathrm{ra}^{-/-}$mice were adoptively transferred to RIPGP.Rag ${ }^{-/-}$recipients. While splenocytes from LCMV-infected control mice induced T1D in RIP-GP.Rag1 ${ }^{-/}$recipients, cells from $\mathrm{Il}_{15 \mathrm{ra}^{-/}}$mice failed to induce disease (Figure 3a), indicating that antigen-experienced LCMV-GP-specific CD8 ${ }^{+}$ $\mathrm{T}$ cells need IL-15R $\alpha$ to sustain pathogenic potential.

In an early study before the discovery of IL-15 transpresentation, Schluns et al. reported using both $I l 15^{-/}$and Ill $5 \mathrm{ra}^{-/}$mice that IL-15R $\alpha$ but not IL-15 is dispensable for activating $\mathrm{CD} 8{ }^{+} \mathrm{T}$ cells following vesicular stomatitis virus infection. ${ }^{12}$ This study concluded that primary antiviral response may not require IL-15 due to low expression of IL-15r $\alpha$ on naive $\mathrm{CD}^{+} \mathrm{T}$ cells, whereas activated cells expressing higher levels of IL-15R $\alpha$ are dependent on it to generate memory cells and their maintenance. The inability of diabetogenic $\mathrm{CD} 8^{+} \mathrm{T}$ cells from LCMV-infected $\mathrm{Il}_{15 \mathrm{ra}^{-/}}$mice to induce disease in RIP-GP. $R a g 1^{-1-}$ recipients (Figure 3a) is consistent with the requirement of IL-15R $\alpha$ to maintain virus-induced memory $\mathrm{CD} 8^{+} \mathrm{T}$ cells. However, it stands in sharp contrast to the ability of splenocytes from diabetic NOD.Il15 $\mathrm{ra}^{-/-}$mice to transfer disease to NOD.scid recipients (Figure 1d). The possibility that IL-15R $\alpha$ expressed by recipient cells might have complemented its absence on donor $\mathrm{CD}^{+} \mathrm{T}$ cells in the NOD model is untenable because this was not the case in RIP-GP model (Figure $3 \mathrm{a}$ and d). The possibility of differential CD4 help from within the donor cell population is also not plausible because both donors harboured a polyclonal a Recipients : RIP-GP.Rag 1-/ Donors : (LCMV infected)
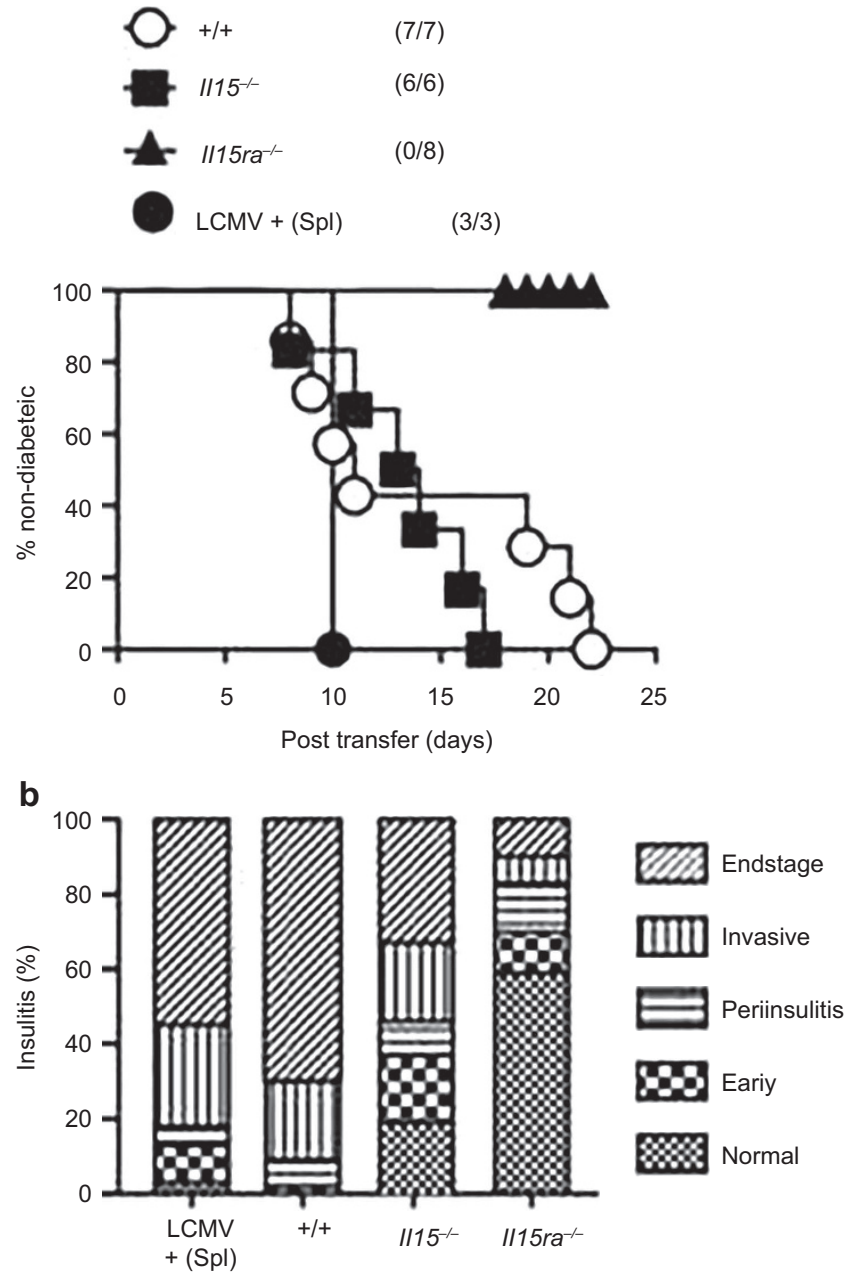

Figure 3 Splenocytes from LCMV-infected IL-15R $\alpha$-deficient mice do not transfer T1D. (a) Splenocytes $\left(10^{7}\right.$ cells) from control, $/ 115^{-1}$, or $1 / 15 \mathrm{ra}^{-/-}$mice infected with LCMV, obtained on day 14 post-infection, were adoptively transferred to RIP-GP.Rag1 ${ }^{-/}$recipients. One group of recipients received LCMV along with splenocytes from uninfected mice (Spl). Infected mice were monitored for T1D. Data pooled from three independent experiments are shown. LCMV+Spl group represent two experiments. (b) Pancreatic sections were assessed for cellular infiltration in the islets and scored.

repertoire. Several factors could contribute in a complex way to the differential requirement for IL-15R $\alpha$ to sustain the pathogenic potential of T cells in these two T1D models. First, diabetogenic $\mathrm{T}$ cells in the NOD model are directed against multiple autoantigens, whereas the target of pathogenic $\mathrm{T}$ cells in the RIPGP model is limited to one epitope, which would limit the severity of the pathogenic process in the latter case. Second, unlike in the case of LCMV infection, where the antigen is eliminated after viral clearance, the autoantigens in the NOD islets are continuously available until the islets are completely destroyed. This implies that the donor cells from diabetic NOD.Il15 $\mathrm{ra}^{-/-}$mice may contain a pool of polyclonal autoreactive $\mathrm{T}$ cells that are still in early stages of activation, whereas GP-reactive cells from 
LCMV-infected have already differentiated into effector and memory lineages, thus requiring IL-15R $\alpha$ to sustain their pathogenicity. Third, the pro-diabetic genetic background might diminish the requirement for IL-15 trans-presentation in maintaining the pathogenic potential of autoreactive T cells.

In contrast to cells from LCMV-infected $\mathrm{Il}_{15 \mathrm{ra}^{-/}}$donors, splenocytes from $I l 15^{-\alpha}$ mice efficiently transferred T1D to RIP-GP. $\mathrm{Ragl}^{-/-}$recipients that were comparable to control cells (Figure $3 a-b)$. This could be explained by the presence of uncleared LCMV from $I l 15^{-}$donors. This notion is supported by rapid induction of T1D in RIP-GP.Rag1 $1^{-/-}$mice by adoptive transfer of splenocytes from uninfected mice along with LCMV (Figure 3).

\section{IL-15 but not IL-15ra is required for antigen cross- presentation to $\mathrm{CD8}^{+} \mathrm{T}$ cells in vivo}

T1D onset in the NOD mouse coincides with shedding of islet autoantigens during physiological tissue remodelling that is taken up by DCs and cross-presented to autoreactive $\mathrm{CD}^{+} \mathrm{T}$ cells in pancreatic draining lymph nodes. ${ }^{32,33}$ Similarly, initiation of the immune response following LCMV or Listeria monocytogenes infections is mediated by the cross-presentation of pathogenderived antigens to $\mathrm{CD}^{+}{ }^{+} \mathrm{T}$ cells. ${ }^{22}$ IL-15 has been implicated in antigen presenting functions of DCs. ${ }^{9}$ To examine the role of IL-15 trans-presentation in antigen cross-presentation to CD8 ${ }^{+}$ T cells, we used LM-OVA. Administration of $10^{3}-10^{4}$ CFU of LM is sufficient to initiate CD $8 \alpha^{+}$DC-mediated cross-presentation of LM-derived peptides. ${ }^{22} \mathrm{CD} 8 \alpha^{+}$DCs also express high levels of IL-15 under steady-state conditions. ${ }^{34}$ As IL-15 or IL-15R $\alpha$ deficiency affects the survival and the numbers of DC under steady state, ${ }^{35}$ we postulated that cross-presentation of systemically administered LM-OVA might be defective in the absence of IL15 trans-presentation. However, our results from the NOD model and the RIP-GP model suggested that IL-15 transpresentation is not required to activate diabetogenic $\mathrm{T}$ cells. To directly test the requirement of IL-15R $\alpha$ in antigen crosspresentation $5 \times 10^{3} \mathrm{CFU}$ of LM-OVA was administered intravenously to wild-type $\mathrm{C} 57 \mathrm{Bl} / 6, \mathrm{Ill} 5^{-1}$ and $I l 15 \mathrm{ra}^{-/}$mice followed by injection of $10^{7}$ CFSE-labelled splenocytes from OT-1 TCR transgenic mice. As expected, OT-1 cells showed robust proliferation in LM-OVA-infected WT mice (Figure 4a). While the injected OT-1 cells failed to proliferate in $I l 15^{-1}$ mice, the same cells underwent efficient proliferation in $\mathrm{Il}_{\mathrm{H}} \mathrm{ra}^{-/}$recipients to the level comparable to that observed in control mice. Proliferation of OT-1 cells in Il15 $\mathrm{ra}^{-/-}$hosts was not due to complementation by IL-15R $\alpha$ expressed on donor OT-1 cells as shown by robust proliferation of $I l 15 \mathrm{ra}^{-/-}$OT-1 cells in $I l 15 \mathrm{ra}^{-/-}$hosts (Figure $4 \mathrm{~b}$ ). These results show that while IL- 15 is essential to mediate antigen cross-presentation to $\mathrm{CD}^{+} \mathrm{T}$ cells, this process does not depend on trans-presentation of IL-15 by IL-15R $\alpha$.

\section{CONCLUSIONS}

While it is well known that IL-15R $\alpha$ is critical for mediating certain functions of IL-15 such as development and maintenance of NK cell and memory $\mathrm{CD}^{+}{ }^{+} \mathrm{T}$ lymphocytes, our findings show that IL-15 can mediate at least some of its key functions without the need for trans-presentation. We have a

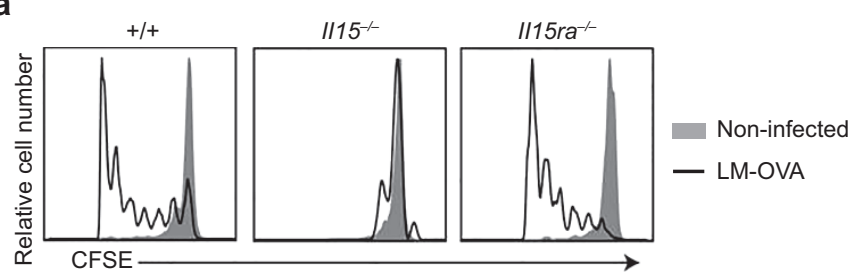

b

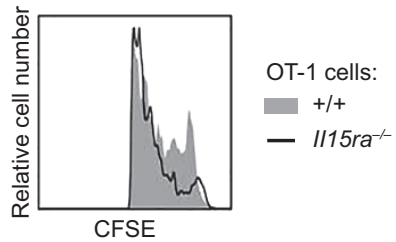

Figure $4 \mathrm{IL}-15$, but not IL-15R $\alpha$ is required for the cross-presentation of LM-OVA antigen in vivo. Wildtype, $/ 115^{-1-}$ or $/ 115 \mathrm{ra}^{-1-}$ mice were injected with $10^{7}$ CFSE labelled lymph node cells from OT-1 (a and $\mathbf{b}$ ) or OT-1.//15ra ${ }^{-1-}$ (b) mice on day 0 . On day 1 the mice received $5 \times 10^{3}$ CFU of LM-OVA intravenously. After 6 days, proliferation was monitored by assessing CFSE fluorescence on gated $\mathrm{CD} 8^{+} \mathrm{T}$ cells. Representative data from three independent experiments with two mice per group, per experiment, are shown.

shown that induction of autoimmune diabetes in two different animal models and viral clearance, which are dependent on IL15 , do not require its trans-presentation by the IL- $15 \mathrm{R} \alpha$ chain. We have also shown that antigen cross-presentation to $\mathrm{CD} 8^{+} \mathrm{T}$ cells, a key process that underlies pathogenesis of T1D and plays an important role in immune response against intracellular pathogens, does not require IL-15R $\alpha$.

Our findings highlight the intricacies of IL-15 signalling that should be considered in therapeutic targeting of the IL-15 signalling pathway. An early study has shown that simian IL-15 can signal via the human IL-15 $\alpha \gamma$ in COS7 cells. ${ }^{36}$ Our data indicate that IL-15R $\alpha$-independent signalling can occur in vivo. During the initiation of the immune response, either in spontaneous T1D or in the clearance of LCMV, IL-15 may primarily act on the non-lymphoid cells to initiate the immune responses. Consistent with this prediction, we have shown that the inhibition of IL-15 signalling using anti-CD122 mAb Tm $\beta 1$ targeting IL-15R $\alpha$ during a short-time window prior to disease onset attenuated disease development in the NOD mouse. ${ }^{16}$ It is also possible that IL-15 may utilize a receptor protein other than IL-15R $\alpha$ to engage the IL-15 $\alpha \gamma$ complex on antigen-presenting cells as shown in mast cells. ${ }^{37}$ Characterization of the IL-15 signalling machinery in non-lymphocytes, particularly antigen-presenting cells, will contribute to a better understanding of the IL-15 signalling and could lead to new approaches for its therapeutic targeting in autoimmune diseases.

\section{ACKNOWLEDGEMENTS}

This work was funded by NSERC discovery grant to S.R. and S. I. D.B. is a recipient of post-doctoral fellowship from FRQS. The authors thank Dr P. Ohashi for the generous gift of the LCMV strain, and Dr Y.G. Chen for critical reading of the 
manuscript. We thank Ms. Y. Cepero-Donates for providing technical help to carry out experiments with Listeria.

\section{CONFLICT OF INTEREST}

The authors declare no conflict of interest.

1 Lodolce J, Burkett P, Koka R, Boone D, Chien M, Chan F et al. Interleukin-15 and the regulation of Iymphoid homeostasis. Mol Immunol 2002; 39: 537-544.

2 Waldmann TA, Tagaya Y. The multifaceted regulation of interleukin15 expression and the role of this cytokine in NK cell differentiation and host response to intracellular pathogens. Annu Rev Immunol 1999; 17: 19-49.

3 Grabstein KH, Eisenman J, Shanebeck K, Rauch C, Srinivasan S, Fung $V$ et al. Cloning of a T cell growth factor that interacts with the beta chain of the interleukin-2 receptor. Science 1994; 264: 965-968.

4 Cui G, Hara T, Simmons S, Wagatsuma K, Abe A, Miyachi H et al. Characterization of the IL-15 niche in primary and secondary lymphoid organs in vivo. Proc Natl Acad Sci U S A 2014; 111: 1915-1920.

5 Tagaya Y, Kurys G, Thies TA, Losi JM, Azimi N, Hanover JA et al. Generation of secretable and nonsecretable interleukin 15 isoforms through alternate usage of signal peptides. Proc Natl Acad Sci U S A 1997; 94: 14444-14449.

6 Lodolce JP, Burkett PR, Boone DL, Chien M, Ma A. T cell-independent interleukin 15Ralpha signals are required for bystander proliferation. J Exp Med 2001; 194: 1187-1194.

7 Schluns KS, Stoklasek T, Lefrancois L. The roles of interleukin-15 receptor alpha: trans-presentation, receptor component, or both? Int J Biochem Cell Biol 2005; 37: 1567-1571.

8 Mortier E, Advincula R, Kim L, Chmura S, Barrera J, Reizis B et al. Macrophage- and dendritic-cell-derived interleukin- 15 receptor alpha supports homeostasis of distinct CD8 + T cell subsets. Immunity 2009; 31: 811-822.

9 Ohteki T, Suzue K, Maki C, Ota T, Koyasu S. Critical role of IL-15-IL$15 \mathrm{R}$ for antigen-presenting cell functions in the innate immune response. Nat Immunol 2001; 2: 1138-1143.

10 Mortier E, Bernard J, Plet A, Jacques Y. Natural, proteolytic release of a soluble form of human IL-15 receptor alpha-chain that behaves as a specific, high affinity IL-15 antagonist. J Immunol 2004; 173 : 1681-1688.

11 Stonier SW, Schluns KS. Trans-presentation: a novel mechanism regulating IL-15 delivery and responses. Immunol Lett 2010; 127: 85-92.

12 Schluns KS, Williams K, Ma A, Zheng XX, Lefrancois L. Cutting edge: requirement for IL-15 in the generation of primary and memory antigen-specific CD8 T cells. J Immunol 2002; 168: 4827-4831.

13 Becker TC, Wherry EJ, Boone D, Murali-Krishna K, Antia R, Ma A et al. Interleukin 15 is required for proliferative renewal of virus-specific memory CD8 T cells. J Exp Med 2002; 195: 1541-1548.

14 Wherry EJ, Becker TC, Boone D, Kaja MK, Ma A, Ahmed R. Homeostatic proliferation but not the generation of virus specific memory CD8 T cells is impaired in the absence of IL-15 or IL15Ralpha. Adv Exp Med Biol 2002; 512: 165-175.

15 Waldmann TA. The biology of IL-15: implications for cancer therapy and the treatment of autoimmune disorders. J Investig Dermatol Symp Proc 2013; 16: S28-S30.

16 Bobbala D, Chen XL, Leblanc C, Mayhue M, Stankova J, Tanaka T et al. Interleukin-15 plays an essential role in the pathogenesis of autoimmune diabetes in the NOD mouse. Diabetologia 2012; 55: 3010-3020.

17 Chen J, Feigenbaum L, Awasthi P, Butcher DO, Anver MR, Golubeva YG et al. Insulin-dependent diabetes induced by pancreatic beta cell expression of IL-15 and IL-15Ralpha. Proc Natl Acad Sci U S A 2013; 110: 13534-13539.

18 Ohashi PS, Oehen S, Buerki K, Pircher H, Ohashi CT, Odermatt B et al. Ablation of "tolerance" and induction of diabetes by virus infection in viral antigen transgenic mice. Cell 1991; 65: 305-317.
19 Ramanathan S, Dubois S, Chen XL, Leblanc C, Ohashi PS, Ilangumaran $S$ et al. Exposure to IL-15 and IL-21 enables autoreactive CD8 T cells to respond to weak antigens and cause disease in a mouse model of autoimmune diabetes. J Immunol 2011; 186: 5131-5141.

20 Ramanathan S, Dubois S, Gagnon J, Leblanc C, Mariathasan S, Ferbeyre $\mathrm{G}$ et al. Regulation of cytokine-driven functional differentiation of CD8 T cells by suppressor of cytokine signaling 1 controls autoimmunity and preserves their proliferative capacity toward foreign antigens. J Immunol 2010; 185: 357-366.

21 Battegay M, Cooper S, Althage A, Bänziger J, Hengartner H, Zinkernagel $\mathrm{RM}$ et al. Quantification of lymphocytic choriomeningitis virus with an immunological focus assay in 24- or 96-well plates. J Virol Methods 1991; 33: 191-198.

22 Belz GT, Shortman K, Bevan MJ, Heath WR. CD8alpha+ dendritic cells selectively present $\mathrm{MHC}$ class I-restricted noncytolytic viral and intracellular bacterial antigens in vivo. J Immunol 2005; 175: 196200.

23 Kennedy MK, Glaccum M, Brown SN, Butz EA, Viney JL, Embers M et al. Reversible defects in natural killer and memory CD8 T cell lineages in interleukin 15-deficient mice. J Exp Med 2000; 191: 771-780.

24 Sandau MM, Kohlmeier JE, Woodland DL, Jameson SC. IL-15 regulates both quantitative and qualitative features of the memory CD8 T cell pool. J Immunol 2010; 184: 35-44.

25 Conlon KC, Lugli E, Welles HC, Rosenberg SA, Fojo AT, Morris JC et al. Redistribution, hyperproliferation, activation of natural killer cells and CD8 T cells, and cytokine production during first-in-human clinical trial of recombinant human interleukin-15 in patients with cancer. J Clin Oncol 2015; 33: 74-82.

26 Seay K, Church C, Zheng JH, Deneroff K, Ochsenbauer C, Kappes JC et al. In vivo activation of human NK cells by treatment with an interleukin-15 superagonist potently inhibits acute in vivo HIV-1 infection in humanized mice. J Virol 2015; 89: 6264-6274.

27 Oh S, Perera LP, Terabe M, Ni L, Waldmann TA, Berzofsky JA et al. IL15 as a mediator of CD4+ help for CD8 + T cell longevity and avoidance of TRAIL-mediated apoptosis. Proc Natl Acad Sci U S A 2008; 105: 5201-5206.

28 Oldstone MB, Nerenberg M, Southern P, Price J, Lewicki H. Virus infection triggers insulin-dependent diabetes mellitus in a transgenic model: role of anti-self (virus) immune response. Cell 1991; 65: 319-331.

29 Verbist KC, Field MB, Klonowski KD. Cutting edge: IL-15independent maintenance of mucosally generated memory CD8 $T$ cells. J Immunol 2011; 186: 6667-6671.

30 Bhadra R, Khan IA. IL-7 and IL-15 do not synergize during CD8 T cell recall response against an obligate intracellular parasite. Microbes Infect 2012; 14: 1160-1168.

31 Lazarevic V, Yankura DJ, DiVito SJ, Flynn JL. Induction of Mycobacterium tuberculosis-specific primary and secondary T-cell responses in interleukin-15-deficient mice. Infect Immun 2005; 73: 2910-2922.

32 Yang Y, Santamaria P. Lessons on autoimmune diabetes from animal models. Clin Sci (Lond) 2006; 110: 627-639.

33 Zhang Y, O'Brien B, Trudeau J, Tan R, Santamaria P, Dutz JP et al. In situ beta cell death promotes priming of diabetogenic CD8 T lymphocytes. J Immunol 2002; 168: 1466-1472.

34 Colpitts SL, Stoklasek TA, Plumlee CR, Obar JJ, Guo C, Lefrançois L et al. Cutting edge: the role of IFN-alpha receptor and MyD88 signaling in induction of IL-15 expression in vivo. J Immunol 2012; 188: 2483-2487.

35 Dubois SP, Waldmann TA, Muller JR. Survival adjustment of mature dendritic cells by IL-15. Proc Natl Acad Sci U S A 2005; 102: 86628667.

36 Anderson DM, Kumaki S, Ahdieh M, Bertles J, Tometsko M, Loomis A et al. Functional characterization of the human interleukin-15 receptor alpha chain and close linkage of IL15RA and IL2RA genes. J Biol Chem 1995; 270: 29862-29869.

37 Tagaya $Y$, Burton JD, Miyamoto $Y$, Waldmann TA. Identification of a novel receptor/signal transduction pathway for IL-15/T in mast cells. EMBO J 1996; 15: 4928-4939. 